\title{
PLANTAS TÍPICAS DO CERRADO BRASILEIRO USADAS COMO INIBIDORES DA \\ ACETILCOLINESTERASE: UMA REVISÃO SISTEMÁTICA
}

Typical Brazilian Cerrado Plants Used as Acetylcholinesterase Inhibitors: A Systematic Review

Plantas típicas de Cerrado brasileño utilizadas como inhibidores de la acetilcolinesterasa: una revisión sistemática

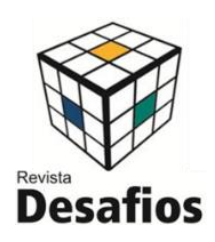

Artigo Original

Original Article

Artículo Original

\section{Rachel de Moura Nunes Fernandes ${ }^{1}$, Elisandra Scapin ${ }^{* 2}$}

${ }^{1}$ Programa de Pós-Graduação em Biodiversidade e Biotecnologia - BIONORTE, Universidade Federal do Tocantins, Palmas, Brasil.

${ }^{2}$ Curso de Engenharia Ambiental, Programa de Pós-Graduação em Ciências do Ambiente - CIAMB, Programa de Pós-Graduação em Biodiversidade e Biotecnologia - BIONORTE, Universidade Federal do Tocantins, Palmas, Brasil.

*Correspondência: Av. NS 15, 109 Norte, Laboratório de Química, Bloco 2, Sala 01, Palmas, Tocantins, Brasil. CEP:77.010-090.e-mail: scapin@uft.edu.br.

\section{RESUMO}

O bioma Cerrado vem se destacando quanto à fonte de compostos bioativos devido à sua flora vegetal, estimada em cerca de sete mil espécies. O uso de plantas para o tratamento de doenças é tão antigo quanto à espécie humana e essa utilização saiu do âmbito do empirismo e hoje ganha status de ciência. Dentre as doenças com maiores índices de crescimento da taxa de mortalidade destacamos a Doença de Alzheimer (DA), a qual está associada ao "déficit" nos níveis de neurotransmissores cerebrais, dentre eles a acetilcolina, responsável pela memória e raciocínio lógico. Os medicamentos modernos elevam os níveis desse neurotransmissor pela inibição da enzima acetilcolinesterase (AChE). Atualmente, as pesquisas buscam novos inibidores da AChE a partir de extratos de plantas. Neste estudo foi realizada uma busca bibliográfica em periódicos especializados nas bases Scientific Eletronic Library Online, Science Direct e Google Acadêmico,utilizando-se as seguintes palavras-chaves: atividade anticolinesterásica e Cerrado. Como resultado obteve-se 32 estudos que após serem submetidos à análise dos títulos possibilitaram a recuperação de 20 trabalhos publicados entre 2009 e 2019. Ao todo foram identificados estudos com 33 famílias botânicas, sendo que a família Fabaceae foi à majoritária. Contabilizou-se 66 espécies analisadas, sendo que $62 \%$ apresentaram atividade anticolinesterásica.

Palavras-chave: Plantas medicinais. Cerrado. Acetilcolinesterase.

\section{ABSTRACT}

The Cerrado biome has been highlighting the source of bioactive compounds due to its plant flora, estimated at about seven thousand species. The use of plants for the treatment of disease is as old as the human species, and its use has gone beyond the scope of empiricism and today it has gained scientific status. Among the diseases with the highest rates of growth in the mortality rate, we highlight Alzheimer's disease (AD), which is associated with a "deficit" in brain neurotransmitter levels, including acetylcholine, responsible for memory and logical reasoning. Modern drugs raise the levels of this neurotransmitter by inhibiting the enzyme acetylcholinesterase (AChE). Research is currently seeking new AChE inhibitors from plant extracts. In this study a bibliographic search was performed in specialized 
journals in the Scientific Electronic Library Online, Science Direct and Google Scholar databases, using the following keywords: anticholinesterase activity and Cerrado. As a result, 32 studies were obtained which, after being submitted to title analysis, allowed the recovery of 20 papers published between 2009 and 2019. In all, studies with 33 botanical families were identified, with the Fabaceae family being the majority. Sixty six species were accounted for, and $62 \%$ showed anticholinesterase activity.

Keywords: Medicinal plants. Thick. Acetylcholinesterase.

\section{RESUMEN}

El bioma Cerrado ha estado destacando la fuente de compuestos bioactivos debido a su flora vegetal, estimada en aproximadamente siete mil especies. El uso de plantas para el tratamiento de enfermedades es tan antiguo como la especie humana, y su uso ha ido más allá del alcance del empirismo y hoy ha adquirido un estatus científico. Entre las enfermedades con las tasas más altas de crecimiento en la tasa de mortalidad, destacamos la enfermedad de Alzheimer (EA), que se asocia con un "déficit" en los niveles de neurotransmisores cerebrales, incluida la acetilcolina, responsable de la memoria y el razonamiento lógico. Las drogas modernas aumentan los niveles de este neurotransmisor al inhibir la enzima acetilcolinesterasa (AChE). La investigación está buscando nuevos inhibidores de AChE a partir de extractos de plantas. En este estudio se realizó una búsqueda bibliográfica en revistas especializadas en las bases de datos de la Biblioteca Electrónica Científica en línea, Science Direct y Google Scholar, utilizando las siguientes palabras clave: actividad anticolinesterasa y Cerrado. Como resultado, se obtuvieron 32 estudios que, después de ser sometidos al análisis del título, permitieron la recuperación de 20 artículos publicados entre 2009 y 2019. En total, se identificaron estudios con 33 familias botánicas, siendo la familia Fabaceae la mayoría. Se contabilizaron 66 especies y el $62 \%$ mostró actividad anticolinesterasa.

Descriptores: Plantas medicinales. Cerrado Acetilcolinesterasa.

\section{INTRODUÇÃO}

O Bioma Cerrado apresenta um cenário exuberante de diversidade biológica, não apenas pela influência dos ecossistemas circundantes (Amazônico, Semi-Árido, Mata Atlântica e Pantanal) bem como pelo arcabouço cultural das populações que o habitam (VILA VERDE et al., 2003). Devido à forma desenfreada de ocupação, aos investimentos na monocultura intensiva de grãos e a pecuária extensiva de baixa tecnologia, $80 \%$ do Cerrado já foi modificado, o que explica os elevados impactos ambientais sofridos pelo segundo maior Bioma do Brasil (MITTERMEIER et al., 2004).

Como uma das formas de limitar a expansão dessa degradação deve-se abrir espaço para projetos que fazem uso da diversidade biológica do Cerrado de forma sustentável. O Cerrado é composto por onze tipos principais de vegetação, enquadrados em formações florestais (Mata Ciliar, Mata de Galeria, Mata Seca e Cerradão), savânicas (Cerrado sentido restrito, Parque de Cerrado, Palmeiral e Vereda) e campestres (Campo Sujo, Campo Limpo e Campo Rupestre), podendo estender para 25 tipos de vegetação, se analisados os subtipos (MMA, 2006).

A indicação de plantas como tratamento medicinal advém do acervo empírico, principalmente de comunidades indígenas e quilombolas, e auxilia no levantamento do patrimônio genético que precisa ser conhecido e absorvido por essa geração para que possa ser utilizado pelas gerações futuras. Os primeiros relatos da utilização das plantas com finalidades terapêuticas datam de 3.000 anos A.C. na China e, apesar do enorme avanço tecnológico presenciado no Século XXI, a cultura sobre o uso de plantas medicinais ainda é o recurso terapêutico de muitas comunidades e grupos étnicos. Silva e Fernandes Junior (2010) constataram que " $80 \%$ da população brasileira consomem apenas $37 \%$ dos medicamentos comercialmente disponíveis, dependendo quase exclusivamente de medicamentos de origem natural".

Hoje, os fitoterápicos são vistos como uma forma saudável de prevenção, tratamento e cura de 
determinadas doenças, tudo isso alavancado em consequência da dependência que os produtos sintéticos vêm ocasionando.

A galantamina é um exemplo claro de tratamento terapêutico utilizando plantas medicinais. É um alcaloide, isolado de plantas da família Amaryllidaceae, utilizado no tratamento da Doença de Alzheimer (DA). Esta enfermidade atinge a memória e a capacidade de raciocínio, devido a seus efeitos neurodegenerativos, afetando diretamente o sistema colinérgico. Esse sistema é responsável pela contração dos músculos lisos, dilatação dos vasos sanguíneos e regulação da taxa de batimentos cardíacos (VIEGAS JUNIOR et al., 2004).

O bom funcionamento do sistema colinérgico depende de níveis normais de uma substância chamada acetilcolina (ACh), um transmissor envolvido no aprendizado e na memória (VINUTHA et al., 2007). Este neurotransmissor é encontrado no cérebro e junções neuromusculares e a hidrólise da acetilcolina é essencial para que as transmissões sinápticas colinérgicas ocorram antes da chegada de um novo impulso elétrico (SANTOS, 2009). Esse processo é realizado pela enzima acetilcolinesterase $(\mathrm{AChE})$, que no sistema nervoso, age como mediadora das sinapses.

Os pacientes com DA apresentam baixos níveis de acetilcolina, devido à elevada atividade da enzima AChE. O tratamento indicado para esses pacientes é fazer uso de inibidores de $\mathrm{AChE}(\mathrm{OH}$ et al., 2004; JUNG e PARK, 2007), o que melhora sintomas cognitivos, comportamentais e funcionais, devido ao retardamento da degradação da ACh, assim o neurotransmissor passa mais tempo na fenda sináptica, intensificando desta forma a transmissão colinérgica (MOTA et al., 2012; OLIVEIRA et al., 2017).

Os atuais inibidores da AChE retardam a degradação metabólica da acetilcolina, otimizando a disponibilidade deste substrato para a comunicação entre as células. Isto auxilia no retardo da progressão da disfunção cognitiva e pode ser eficaz para alguns pacientes nos estágios inicial e intermediário da doença, entretanto, os efeitos colaterais como náuseas, vômitos, diarreia, perda de peso, tontura, dor de cabeça e cansaço, são relatados constantemente (ANAND e SINGH, 2013).

A taxa de mortalidade referente à Doença de Alzheimer vem aumentando significativamente em todo mundo nas duas últimas décadas. No Brasil, segundo Teixeira et al. (2015), as taxas de mortalidade nos idosos com 60 anos ou mais, referentes à DA, tem aumento anual e significativo. Nos Estados Unidos a taxa aumentou em 55\% entre os anos 1999 e 2014 e entre as dez principais causas de morte em idosos destaca-se a taxa ajustada para a doença de Alzheimer, com variação percentual de 43\% (MOSCHETTI et al., 2012).

Nessa perspectiva, várias espécies de plantas vêm sendo exploradas na busca de inibidores mais seletivos e com menos efeitos colaterais para $\mathrm{o}$ tratamento e cura da DA. Assim, este estudo teve como objetivo realizar o levantamento das espécies de plantas do Cerrado que foram analisadas frente à atividade anticolinesterásica.

\section{MATERIAIS E MÉTODOS}

Este trabalho configurou-se em um levantamento de literatura sobre a atividade anticolinesterásica realizada com extratos obtidos a partir de plantas típicas do Cerrado. Foi realizada uma busca bibliográfica em periódicos especializados nas bases Scientific Eletronic Library Online, Science Direct e Google Acadêmico, utilizando-se as seguintes palavras-chaves: atividade anticolinesterásica e Cerrado nos idiomas português e inglês. Como resultado obteve-se 32 estudos que após serem submetidos à análise possibilitaram a avaliação de 20 trabalhos publicados entre 2009 e 2019. 


\section{RESULTADOS E DISCUSSÃO}

Após análise dos trabalhos pôde-se verificar que houve estudos nos estados de Goiás, Minas Gerais, Piauí, Maranhão, Roraima, Paraná, Tocantins, Mato Grosso do Sul, São Paulo e no Distrito Federal. Os estados majoritários foram Goiás e Minas Gerais, com $20 \%$ dos estudos cada, seguido de $15 \%$ do Distrito Federal, conforme demonstrado na Figura 1.

Figura 1. Número de trabalhos publicados por estado da região do Cerrado.

\section{TRABALHOS}

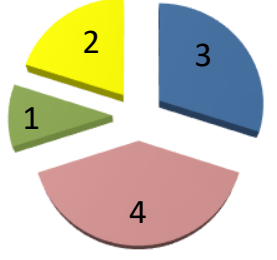

DF

GO; MG

MA; PR; RR; SP;TO

$\mathrm{Pl} ; \mathrm{MS}$

No total 33 famílias botânicas foram

foi a mais citada, com 9 espécies, correspondendo a identificadas (Figura 2), sendo que a família Fabaceae

$14 \%$ das plantas. Outras que também se destacaram
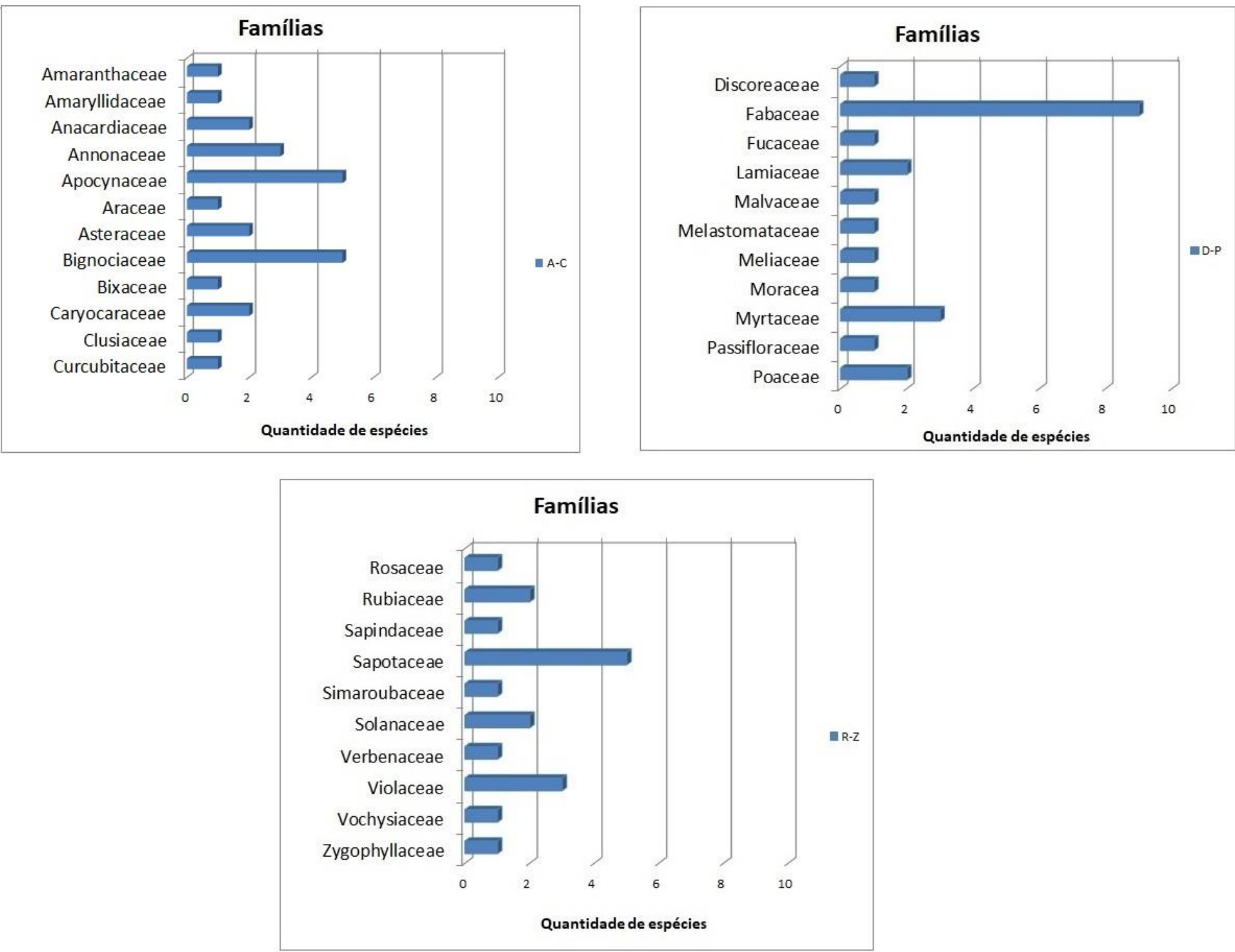
foram a Apocynaceae, Bignoniaceae e Sapotaceae com com 5\%. As demais espécies não ultrapassaram 3\% de 6\% ambas, e a Annonaceae, Myrtaceae e Violaceae citação.

Figura 2. Quantidade de espécies (em ordem alfabética) por família citada nos estudos.

Na Tabela 1 estão denominadas (nome científico) as 66 espécies vegetais citadas, seus nomes comuns e a resposta à atividade anticolinesterásica.

Tabela 1. Relação das plantas do bioma Cerrado testadas para atividade anticolinesterásica.

\begin{tabular}{|c|c|c|}
\hline & & \\
\hline Nome Científico & Nome Comum & $\overline{\mathbf{A A}}$ \\
\hline Ageratum conyzoide & Erva de são joão & - \\
\hline Allamanda blanchetti & Viúva alegre & - \\
\hline Anadenanthera peregrina & Angico & - \\
\hline Anchietea pyrifolia & Cipó sumo & + \\
\hline Annona crassiflora & Araticum & + \\
\hline Annona coriacea & Fruta do conde & + \\
\hline Annona cacans & Araticum cagão & + \\
\hline Arundo donax & Cana comum & + \\
\hline Bauhinia forficata & Pata de vaca & - \\
\hline Bauhinia microstachya & Escada de macaco & + \\
\hline Caryocar cf. villosum & Pequiarana & - \\
\hline Caryocar brasiliense & Pequi & + \\
\hline Cochlospermum regium & $\begin{array}{l}\text { Algodãozinho do } \\
\text { campo }\end{array}$ & + \\
\hline Copaifera langsdorffii & Copaíba & + \\
\hline Discorea glandulosa & Japecanga & + \\
\hline Eugenia dysenterica & Cagaita & + \\
\hline Fucus vesicolosus & Fava do mar & + \\
\hline Garcinia cambogia & Malabar tamarindo & - \\
\hline Genipa americana & Genipapo & - \\
\hline Hancornia speciosa & Mangaba & + \\
\hline Handroanthus ochraceus & Ipê amarelo & + \\
\hline Handroanthus serratifolius & Ipê amarelo & - \\
\hline Hippeastrum goianum & Amarílis & + \\
\hline Himatanthus lancifolius & Agoniada & + \\
\hline Hybanthus bigibbosus & NI & + \\
\hline Hymenaea stignocarpa & Jatobá & - \\
\hline Hyptis dilatata & Hortelã do mato & + \\
\hline Jacaranda micrantha & Caroba-rosa & + \\
\hline Jacaranda sp. & Caroba & + \\
\hline Jacaranda oxyphylla & NI & + \\
\hline Lippia origanoide & Alecrim-pimenta & + \\
\hline Lychnophora pinaster & Arnica & - \\
\hline Mangifera indica & Mangueira & + \\
\hline
\end{tabular}




\begin{tabular}{|c|c|}
\hline Morus nigra & Amora negra \\
\hline Mouriri elliptica & Croadinha, Puçá \\
\hline Myracrodruon urundeuva & Aroeira \\
\hline Passiflora actinia & Maracujá \\
\hline Pfaffia paniculata & Ginseng-brasileiro \\
\hline Philodendron sellowianum & Cipó imbé \\
\hline Phoradendron falcifrons & NI \\
\hline Picrasma crenata & Pau amargo \\
\hline Plathymenia reticulata & Vinhático \\
\hline Plectranthus neochilus & Boldo japonês \\
\hline Pouteria caimito & Abieiro \\
\hline Pouteria gardneri & Sapotinha \\
\hline Pouteria ramiflora & Curriola \\
\hline Pouteria torta & Abiu amarelo \\
\hline Psidium guajava & Goiaba-araçá \\
\hline Pterodon pubescens & Sucupira branca \\
\hline Qualea parviflora & Pau-terra \\
\hline Rauvolfia sellowii & Café de anta \\
\hline Rosa chinensis & Rosa branca \\
\hline Rudgea viburnoides & Casca-branca \\
\hline Sapindus saponaria & Jequiri \\
\hline Serjania erecta & Cipó cinco-folhas \\
\hline Solanum lycocarpum & Lobeira \\
\hline Solanum scuticum & Jurubeba \\
\hline Stryphnodendron adstringens & Barbatimão verdadeiro \\
\hline Stryphnodendron coriaceum & Barbatimão \\
\hline Syzygium aromaticum & Cravo da india \\
\hline Tabernaemontana solanifolia & Leiteira \\
\hline Tribulus terrestris & Abre os olhos \\
\hline Trichilia catigua & Catiguá \\
\hline Uuehea divaricata & Açoita cavalo \\
\hline Wilbrandia ebracteata & Tauiá \\
\hline Zea mays & Milho \\
\hline
\end{tabular}

AA - Atividade Anticolinesterásica; (+) indica teste positivo; (-) indica teste negativo; NI - nome comum não identificado

No estado de Goiás, a espécie Mouriri elliptica (Croadinha, Puçá) apresentou extratos com inibição relevante, acima de $70 \%$. Foram analisados os extratos brutos de acetato de etila e de metanol e frações desses extratos. As frações foram obtidas após $10 \mathrm{~g}$ do extrato bruto ser solubilizado (em água para o metanol e em metanol para o acetato de etila) e aplicado em uma coluna cromatográfica $(45 \mathrm{~cm} \times 5,5 \mathrm{~cm})$ empacotada com Sephadex LH20. Destacam-se duas frações metanólicas que apresentaram uma concentração de inibição de 50\% ( $\left.\mathrm{IC}_{50}\right)$ correspondente a 4,68 e 4,18 $\mu \mathrm{g} / \mathrm{mL}$, resultados satisfatórios quando comparados à Eserina (IC $\left.{ }_{50} 1,37 \mu \mathrm{g} / \mathrm{mL}\right)$ (SILVA, 2016).

As frações do extrato metanólico da espécie Qualea parviflora (Pau-terra) e Rudgea viburnoides (Casca-branca) também foram testadas como inibidores enzimáticos para $\mathrm{AChE}$, sendo que o extrato 
metanólico da espécie Rudgea, apresentou maior inibição frente à enzima (SILVA et al., 2018).

O estudo de Santos et al. (2015) pesquisaram as sementes de quatro frutas comestíveis do Cerrado brasileiro: Annona crassiflora (Araticum), Eugenia dysenterica (Cagaita), Hymenaea stignocarpa (Jatobá) e Hancornia speciosa (Mangaba), mas não foi detectada atividade inibitória sobre acetilcolinesterase em nenhum dos extratos das sementes estudadas.

Porfiro (2017) analisou a espécie Pterodon pubescens, nativa do Cerrado e conhecida popularmente como Sucupira branca. O autor obteve os extratos brutos em metanol e em acetato de etila e também frações desses extratos, sendo que cada extrato bruto foi fracionado em apenas uma fase com o solvente oposto supracitado. Os resultados de inibição obtidos para a $P$. pubescens foram valores inibitórios de $73 \%, 77 \%, 79 \%$ e $70 \%$, respectivamente, na dose recomendada de $80 \mu \mathrm{g} / \mathrm{mL}$, não havendo diferença significativa de inibição entre os extratos brutos e as fases.

Das espécies analisadas no estado de Minas Gerais, Mota et al. (2011) selecionaram as plantas Ageratum conyzoides (Erva de São João.), Lychnophora pinaster (Arnica), Rosa chinensis (Rosa branca), Solanum scuticum (Jurubeba) e Solanum lycocarpum (Lobeira) e destas foram feitos extratos etanólicos das partes aéreas e/ou raízes. Dentre as espécies analisadas, a espécie $S$. lycocarpum mostrou ser a melhor fonte de inibição da acetilcolinesterase, tendo como controle positivo a galantamina. A espécie R. chinesis, o exemplar da família Rosaceae testado, apresentou atividade positiva e as plantas da família Asteraceae não apresentaram atividade.

A espécie Jacaranda sp., conhecida como Caroba, foi avaliada através dos extratos etanólicos das três partes da planta (folhas, galhos e raízes) e apresentou grande capacidade de inibição da enzima acetilcolinesterase, com ênfase nos extratos obtidos dos galhos (CAMPOS et al., 2011). Resultados semelhantes foram obtidos por Pereira et al. (2016) com extratos hexânicos dos galhos da espécie Jacaranda oxyphylla.

Estudos realizados por Carvalho (2017) identificaram que o óleo essencial de Lippia origanoide, o Alecrim-pimenta, apresenta elevado potencial inibidor da enzima acetilcolinesterase, com $\mathrm{IC}_{50}=1,81 \mu \mathrm{g} / \mathrm{mL}$, justificado pela presença do composto acetato de farnesila. O carvacrol e o 1,8cineol, compostos constituintes do óleo essencial da $L$. origanoide, também foram avaliados sobre a enzima acetilcolinesterase, sendo que, seus resultados demonstraram inibição relevante, com valores de $\mathrm{IC}_{50}$ iguais a 2,30 e 0,57 $\mu \mathrm{g} / \mathrm{mL}$, respectivamente.

Na região do Distrito Federal, o extrato aquoso das folhas da Eugenia dysenterica (Cagaita) apresentou $66,3 \%$ de inibição da acetilcolinesterase na concentração de $1 \mathrm{mg} / \mathrm{mL}$. O valor de $\mathrm{IC}_{50}$ para o extrato foi $155,20 \mathrm{mg} / \mathrm{mL}$ frente ao padrão fisostigmina 18,69 mg/mL. Após o isolamento dos compostos quercetina e catequina do extrato, os mesmos apresentaram valores de $\mathrm{IC}_{50} 46,59 \mathrm{mg} / \mathrm{mL}$ e 42,39 $\mathrm{mg} / \mathrm{mL}$, respectivamente (GASCA et al., 2017).

Anteriormente ao estudo de GASCA et al.( 2017), Souza (2011) avaliou extratos aquosos, etanólicos e hexânicos da folha, caule e frutos de 14 espécies do bioma Cerrado, sendo Allamanda blanchetti (Viúva alegre), Hancornia speciosa (Mangaba), Tabernaemontana solanifolia (Leiteira), Morus nigra (Amora negra), Eugenia dysenterica (Cagaita), Stryphnodendron adstringens (Barbatimão verdadeiro), Plectranthus neochilus (Boldo japonês), Genipa americana (Genipapo), Pouteria gardneri (Sapotinha), Pouteria ramiflora (Curriola), Pouteria caimito (Abieiro), Pouteria torta (Abiu amarelo), Caryocar cf. (Pequiarana), Sapindus saponaria (jequiri) e nenhum dos extratos exibiu potencial de inibição relevante. 
Ainda no Distrito Federal, Costa et al. (2019) avaliaram o extrato etanólico (previamente desengordurado com hexano) do bulbo da Hippeastrum goiamum (Amarílis), germinada in vitro. Os pesquisadores verificaram uma leve atividade anticolinesterásica do extrato, em função da presença do alcaloide licorina.

No estado do Piauí espécies da família Bignoniaceae, os Ipês, (Handroanthus ochraceus e H. serratifolius), foram utilizados para obtenção de extratos hexânicos, etanólicos e aquosos, sendo que apenas o extrato etanólico das folhas de H. ochraceaus (ipê amarelo) apresentou resultado significativo, equiparando-se a inibição da cafeína (COSTA e ARAUJO, 2011).

As espécies Eugenia dysenterica (Cagaita) e Annona crassiflora (Araticum), também foram estudadas no estado supracitado, e a atividade qualitativa anticolinesterásica do óleo essencial de folhas de E. dysenterica teve resultados promissores de $\mathrm{IC}_{50}=0,916 \mu \mathrm{g} / \mathrm{mL}$ em comparação ao fármaco rivastigmina $\left(\mathrm{IC}_{50}=1,87 \mu \mathrm{g} / \mathrm{mL}\right), \quad$ inibidor convencional da AChE (FEITOSA et al., 2017).

Formagio et al. (2015) realizaram um estudo no Mato Grosso do Sul, com 3 espécies de Annonaceae, Annona crassiflora (Araticum), Annona coriacea (Fruta do conde), Annona cacans (Araticum cagão) e verificaram positiva a atividade anticolinesterásica para os extratos metanólicos das folhas e sementes das espécies citadas.

No mesmo estado, Pedroso et al. (2019) realizaram testes in vivo em ratos, com extrato hidrometanólicos (70\%) da raiz da Cochlospermum regium (Algodãozinho do campo), para verificar a atividade anticolinesterásica da espécie. Os resultados demonstraram inibição da $\mathrm{AChE}$ em diferentes estruturas cerebrais (no córtex cerebral, no hipotálamo e no hipocampo) dos animais, que tiveram administração oral do extrato.
No estado do Maranhão, Penido (2017) selecionou 11 espécies de plantas medicinais, a partir de um estudo anterior, enfatizando os maiores potenciais de atividade antioxidante. Extratos hidroetanólicos (70\%) foram obtidos da casca das espécies Anadenanthera peregrina (Angico); Bauhinia forficata (Pata de vaca); Copaifera langsdorffii (Copaíba); Hancornia speciosa (Mangaba); Myracrodruon urundeuva (Aroeira); Stryphnodendron coriaceum (Barbatimão); da folha da Luehea divaricata (Açoita cavalo); Mangifera indica (Mangueira); Plathymenia reticulata (Vinhático); Psidium guajava (Goiaba-araçá); e do fruto da Syzygium aromaticum (Cravo da índia). Após analisar as atividades antioxidante e inibitória da acetilcolinesterase, o estudo apontou seis espécies com relevantes potenciais de agentes terapêuticos contra a DA: Hancornia speciosa, Myracrodruon urundeuva, Copaifera langsdorffii, Stryphnodendron coriaceum, Psidium guajava, Mangifera indica.

Almeida (2017) realizou um estudo em Roraima analisando os óleos essenciais das folhas e flores da espécie Hyptis dilatata (Hortelã do mato). A avaliação levou em consideração o período chuvoso e seco da região. O óleo essencial extraído das flores, nos dois períodos, inibiu a enzima acetilcolinesterase acima de $90 \%$, enquanto que o óleo essencial extraído das folhas, no período seco, apresentou a maior inibição, 96,4\%. Acredita-se que os compostos monoterpeno fenchona, $\alpha$-pineno, 3-careno, limoneno, cânfora, $\beta$ pineno e $\beta$-cariofileno, presentes no óleo essencial da folha, tenham ação direta na inibição da enzima acetilcolinesterase (ALMEIDA, 2017).

Os testes com Serjania erecta (Cipó cincofolhas) coletadas no estado de São Paulo, indicou que a fração flavonoide do extrato aquoso, apresentou melhor seletividade para inibição da AChE, quando comparada às frações hexânica e acetato de etila (BROGGINI et al., 2010). 
No Paraná, Seidl (2010) avaliou a atividade inibitória de dezenove extratos hidroetanólicos (56\%) de diferentes espécies, tais como Pfaffia paniculata (Ginseng-brasileiro), Himatanthus lancifolius (Agoniada), Rauvolfia sellowii (Café de anta), Tribulus terrestris (Abre os olhos), Philodendron sellowianum (Cipó imbé), Jacaranda micrantha (Caroba-rosa), Fucus vesiculosus (Fava do Mar), Garcinia cambogia (Malabar tamarindo), Wilbrandia ebracteata (Tauiá), Discorea glandulosa (Japecanga), Bauhinia microstachya (Escada de macaco); Trichilia catigua (Catiguá), Passiflora actinia (Maracujá), Zea mays (Milho), Arundo donax (Cana comum), Picrasma crenata (Pau amargo), Anchietea pyrifolia (Cipó sumo), Hybanthus bigibbosus e Phoradendron falcifrons.

Para a espécie Rauvolfia sellowii as frações diclorometano, n-butanol e aquosa foram às mais ativas, enquanto que, para a espécie Himatanthus lancifolius foram às frações diclorometano e acetato de etila. Ao alcaloide uleína, identificado na espécie $H$. lancifolius, foi atribuído $\mathrm{IC}_{50}=0,45 \mu \mathrm{M}$, valor próximo ao intervalo de $\mathrm{IC}_{50}$ referente à galantamina $(0,39$ a 1,5 $\mu \mathrm{M})$. Dentre todos os extratos avaliados, somente as espécies de $P$. actinia, Z. mayz e G. cambogia não apresentaram resultados de inibição da enzima satisfatórios (SEIDL, 2010).

Oliveira et al. (2018) realizaram a pesquisa com extratos hidroetanólicos (70\%) e com frações orgânicas (hexano, clorofórmio, acetato de etila, butanol) da folha do Caryocar brasiliense (Pequi) coletadas no estado do Tocantins. As frações hexânica e clorofórmica não apresentaram atividade anticolinesterásica. Dentre os demais extratos, a fração com acetato de etila obteve a melhor atividade $\left(\mathrm{IC}_{50}=\right.$ $81,5 \mu \mathrm{g} / \mathrm{mL}$ ), valor aproximado ao padrão neostigmina $\left(\mathrm{IC}_{50}=88,0 \mu \mathrm{g} / \mathrm{mL}\right)$.

Assim, observamos após este levantamento que no bioma Cerrado, 36 espécies de plantas possuem potencial de inibição da atividade colinesterásica, sendo 4 espécies coletadas no estado de Goiás, 3 espécies em Minas Gerais, 1 no Distrito Federal, 2 no Piauí, 6 no Maranhão, 1 em Roraima, 1 no Tocantins, 1 em São Paulo, 16 no Paraná e o Pequi no Ceará.

Apesar de o Brasil ser um país biologicamente tão rico, estudos referentes a plantas medicinais cresceram somente a partir dos anos 2005 (CARNEIRO et al., 2014), muito provavelmente, devido à instituição da Política Brasileira de Plantas Medicinais. Carneiro et al. (2014) realizaram um levantamento entre os anos de 1995 a 2011, sobre as tendências dos estudos com plantas medicinais no Brasil e os dados na base Scielo do ano de 2009 se destacaram, totalizando aproximadamente 45 publicações, um crescimento significativo, pois a média anterior ao ano de 2005 era de 1 publicação por ano.

Esses dados demonstram que apesar do interesse no assunto, ainda são ínfimas as pesquisas em uma região com enorme biodiversidade e com ecossistemas tão ameaçados.

Podemos relatar com o nosso levantamento que a Myracrodruon urundeuva é uma das plantas que consta na lista de plantas ameaçadas de extinção conforme Portaria do MMA 443/2014 (MMA, 2014).

Nesse contexto há a necessidade de uma reorganização das estruturas do uso dos recursos naturais, que padronizem planos de manejos, para que se possa compreender o papel desse recurso natural no ecossistema e em consequência viabilizar o seu uso e até mesmo para garantir que futuras gerações usufruam dessas potencialidades.

\section{CONCLUSÃO}

Analisando os dados obtidos podemos afirmar que $62 \%$ das espécies avaliadas apresentaram atividade inibidora da acetilcolinesterase. 
A espécie Eugenia dysenterica apareceu em 3 estudos, embora estes demonstrassem que extratos da semente, das folhas, do caule e dos frutos não apresentaram atividade de inibição, em 2017 novos estudos, no Distrito Federal e no Piauí, tanto o extrato aquoso como o óleo essencial da folha apresentaram inibição da acetilcolinesterase.

Outra espécie também reincidente em estudos foi a Hancornia speciosa, sendo que em Goiás e no Distrito Federal não foram verificados testes positivos para a inibição da acetilcolinesterase, embora no Maranhão, a espécie foi apontada como relevante potencial de agentes terapêuticos contra a DA.

Dados do último levantamento das plantas do Cerrado quantificam 12.734 espécies, das quais 10.845 são plantas pequenas. Estas são responsáveis pela cobertura do solo, prevenindo a erosão causada pelo vento ou pela enxurrada, facilitando a infiltração das chuvas, realizando também o armazenamento de carbono no solo (DURIGAN et al., 2018).

Além da questão de proteção ambiental e do potencial terapêutico, o fator econômico também pode ser relacionado às essas plantas, tanto pelo potencial omamental como pelas propriedades nutritivas.

O levantamento realizado demonstra o potencial das plantas do bioma Cerrado, considerando que poucas espécies foram investigadas, embora apresente resultados in vitro positivos se comparados aos atuais inibidores. Testes in vivo devem ser realizados para confirmar os resultados promissores aqui observados. O isolamento e identificação de novos princípios ativos se faz necessário perante a diversidade de plantas do bioma.

\section{AGRADECIMENTO}

Agradecemos a Universidade Federal do Tocantins pelo apoio.
Todos os autores declararam não haver qualquer potencial conflito de interesses referente a este artigo.

\section{REFERÊNCIAS}

ALMEIDA, S.P. Estudos químicos e biológicos dos óleos essencias e extratos de Hyptis dilatata benth (lamiaceae), procedentes da serra do tepequém amajari / roraima. manaus, AM. Tese de Doutorado. Universidade Federal do Amazonas. Manaus - UFAM; 2017.

ANAND, P.; SINGH, B.; A review on cholinesterase inhibitors for Alzheimer's disease. Archives of Pharmacal Research. v.36, n.4, p.375-399, 2013.

BROGGINI, L. S.C.; FERNANDES, R.S.; NOGUEIRA, T.; SUZANO, F.R.; CAETANO, A.L.; BUCK, H. S.; COUTO, L.B.; FRANÇA, S.C.; PEREIRA, P.S. Behavioral and enzymatic bioassays with Serjania erecta Radlk., Sapindaceae, correlated with cognitive dysfunctions. Rev. Bras. Farmacogn. Braz. J. Pharmacogn. v.20, n.4, p.519-528, 2010.

CAMPOS, K.F.; MOURA, F.; SALLUM, W. S. T.; MOTA, R.F.; TAKAHASHI, J. A. 2011. Estudo do extrato etanólico de Jacaranda sp., uma espécie vegetal do cerrado, como fonte de antibóticos e substâncias para o tratamento do mal de alzheimer. In: Reunião Anual da SBPC, 63, Goiânia, Anais. SBPC, 2011. Disponível em: < http://www.sbpcnet.org.br/livro/63ra/resumos/resumo s/2100.htm>. Acesso em: 23/06/2019.

CARNEIRO, F. M.; SILVA, M. J.P.; BORGES, L. L.; ALBERNAZ, L. C.; COSTA, J. D. P., Tendências dos Estudos com Plantas Medicinais no Brasil. Revista Sapiência: sociedade, saberes e práticas educacionais, v.3, n. 2, p.44-75, 2014.

CARVALHO, S.Y.B. Óleo essencial de Lippia origanoides: avaliação do potencial fungitóxico e estudo do efeito inibitório sobre acetilcolinesterase. São João Del Rei, MG. Monografia de Graduação. Universidade Federal de São João Del Rei; 2017.

COSTA, G. G. P.; SILVA, C. A. G.; GOMES, J.V. D.; TORRES, A. G.; SANTOS, I. R. I.; ALMEIDA, F. T. C.; FAGG, C. W.; SIMEONI, L. A.; SILVEIRA, D.; GOMES- COPELAND, K. K. P. Influence of in vitro micropropagation on lycorine biosynthesis and anticholinesterase activity in Hippeastrum goiamum. Revista Brasileira de Farmacognosia. v.29, n.1, p. 262-265, 2019. 
COSTA, L.P.S.; ARAUJO, M.S.R. 2011. Documentação química e farmacológica de espécies vegetais do cerrado e caatinga piauiense. Disponível em:

http://leg.ufpi.br/21sic/Documentos/RESUMOS/Moda lidade/Exatas/Luis\%20Paulo.pdf $>$. Acesso em: 12/07/2019.

FEITOSA, C. M.; BARBOSA, A. R.; MELO, C. H. S.; FREITAS, R. M.; FONTES, J. E. N.; Costa, E. V.; RASHED, K. N. Z.; COSTA JÚNIOR, J. S. Antioxidant and anticholinesterase activities of the essential oil of Eugenia dysenterica DC. African Journal of Pharmacy and Pharmacology. v. 1, n.19 p. 241-249, 2017.

FORMAGIO, A.S.; VIEIRA, M.C.; VOLOBUFF, C.R.; SILVA, M.S.; MATOS, A.I.; CARDOSO, C.A.; FOGLIO, M.A.; CARVALHO J.E. In vitro biological screening of the anticholinesterase and antiproliferative activities of medicinal plants belonging to Annonaceae.

Braz J Med Biol Res. v.48, n.4, p.308-315, 2015.

GASCA, C. A.; CASTILLO W.O., TAKAHASHI, C.S., FAGG, C.W.; MAGALHÃES, P.O.; FONSECABAZZO, Y.M.; SILVEIRA, D. Assessment of anticholinesterase activity and cytotoxicity of cagaita (Eugenia dysenterica) leaves. Food and Chemical Toxicology, v.109, n.2, p. 996-1002, 2017.

JUNG, M.; PARK, M. Acetylcholinesterase inhibition by flavonoids from Agrimonia pilosa. Molecules, v.12, p.2130- 2139, 2007.

MARQUES, J. P.; LOPES, G.C., Alcaloides como agentes antitumorais: considerações químicas e biológicas. Revista UNINGÁ Review. v.24, n.1, p.5661, 2015.

MINISTÉRIO DO MEIO AMBIENTE - MMA, 2006. Programa Cerrado Sustentável. Disponível em:<http://www.mma.gov.br/estruturas/201/_arquivos /programa_cerrado_sustentvel_201.pdf $>$. Acesso em: 10/06/2018.

MINISTÉRIO DO MEIO AMBIENTE - MMA. Portaria 443 de 17 de dezembro de 2014. Lista nacional das espécies da flora brasileira ameaçadas de extinção. Brasília, 18 dez., Seção 1, p. 110-121, 2014.

MITTERMEIER, R.;ROBLES GIL, P.; HOFFMANN, M.; PILGRIM, J.; BROOKS, T.; MITTERMEIER, C.G.; LAMOREUX, J.; FONSECA, G.A.B. Hotspots revisited: Earth's biologically richest and most endangered terrestrial ecoregions. México. CEMEX/Agrupación Sierra Madre. 392p. 2004.
MOSCHETTI, K.; CUMMINGS , P.L.; SORVILLO, F.; KUO, T. Burden of Alzheimer's disease-related mortality in the United States, 1999-2008. J Am Geriatr Soc, v.60, p.1509-1514, 2012.

MOTA, W.M.; BARROS, M.L.; CUNHA, P.E.L.; SANTANA, M.V.A.; STEVAM, C.S.; LEOPOLDO, P.T.G., FERNANDES, R.P.M., Avaliação da inibição da acetilcolinesterase por extratos de plantas medicinais. Rev. Bras. PI. Med., Botucatu, v.14, n.4, p.624-628, 2012.

MOTA, R. F.; CAMPOS, K.F.; MOURA, F.; SALLUM, W. S. T.; TAKAHASHI, J. A., 2011. Bioprospecção da atividade de plantas medicinais do cerrado das famílias asteraceae, rosaceae e solanaceae como fontes de fármacos inibidores da acetilcolinesterase. In: Reunião Anual da SBPC, 63, Goiânia, Anais Virtuais. SBPC, 2011. Disponível em: <http://www.sbpcnet.org.br/livro/63ra/resumos/resum os/2877.htm>. Acesso em: 01/06/2019.

OH, M.H; HOUGHTON, PJ; WHANG, WK; CHO $\mathrm{JH}$., Screening of Korean herbal medicines used to improve cognitive function for anti-cholinesterase activity. Phytomedicine, v.11, p.544-8, 2004.

OLIVEIRA, L. V.; ANJOS, C.J. F.; CONFESSOR, M.; VILAR D. A.; VILAR, M.S.A. 2017. Fitoterapia como alternativa ao retardamento do alzheimer. Disponível em: http://www.editorarealize.com.br/revistas/conbracis/tr abalhos/TRABALHO_EV071_MD1_SA4_ID1360_0 9052017100412.pdf. Acesso em: 11/08/2019.

OLIVEIRA, T.S.; THOMAZ, D.V.; SILVA NERI, H.F.; CERQUEIRA, L.B.; GARCIA, L.F.; GIL, H.P.V.; PONTAROLO, R.; CAMPOS, F.R.; COSTA, E.A.; SANTOS, F.C.; SOUZA GIL, E.; GHEDINI, P.C. Neuroprotective effect of caryocar brasiliense camb. Leaves is associated with anticholinesterase and antioxidant properties. Oxid Med Cell Longev. v.2018, p.1-12, 2018.

PEDROSO, T. F. M.; BONAMIGO, T. R.; SILVA, J.; VASCONCELOS, P.; FÉLIX, J. M.; CARDOSO, C. A. L.; SOUZA, R. I. C.; SANTOS, A. C.; VOLOBUFFA, C. R. F.; FORMAGIO, A. S. N.; TRICHEZA, V. D. K. Chemical constituents of Cochlospermum regium (Schrank) Pilg. root and its antioxidant, antidiabetic, antiglycation, and anticholinesterase effects in Wistar rats. Biomedicine \& Pharmacotherapy. v.111, p.1383-1392, 2019.

PENIDO, A. B.; MORAIS, S. M.; RIBEIRO, A. B.; ALVES, D. R.; RODRIGUES, A. L. M. S.; L. H; MENEZES, J. E.S. A. Medicinal plants from northeastern Brazil against Alzheimer's disease. 
Evidence-Based Complementary and Alternative Medicine, v.2017, p. 1-7, 2017.

PEREIRA, V. V.; SILVA, R. R.; DOS SANTOS, M.; DIAS, D. F.; MOREIRA, M.; TAKAHASHI, J.A. Antioedematogenic activity, acetylcholinesterase inhibition and antimicrobial properties of Jacaranda oxyphylla. Nat Prod Res. v.30, n.17, p.1974-1979. 2016.

PORFIRO, C.A., Atividade inibitória da enzima acetilcolinesterase frente aos extratos das folhas de Perodon pubescens Benth, Rio Verde, GO. Dissertação de Mestrado. Instituto Federal de Tecnologia Goiano; 2017.

SANO, E.E; ROSA, R, BRITO, JL; FERREIRA LG. , Land cover mapping of the tropical savanna region in Brazil. Environ. Monit. Assess. v.166, p.113-124, 2010.

SANTOS, G.H.S.F.; BATISTA, K. A.; GHEDINI, P.C.; FERNANDES, K.F.; GARCIA-RODRIGUEZ, A. 2015. Inibição Enzimática Por Toxinas De Sementes De Frutos Do Cerrado. Disponível em: https://proceedings.science/slaca/slaca-

2015/papers/inibicao-enzimatica-por-toxinas-de-

sementes-de-frutos-do-cerrado?lang=pt-br Acesso em: julho de 2019.

SANTOS, P. I. M. Avaliação da toxicidade aguda do inseticida metomil e o seu efeito sobre a atividade de acetilcolinesterase do peixe Danio rerio. Natal, RN. Dissertação de Mestrado. Universidade Federal do Rio Grande do Norte; 2009.

DURIGAN, G.; PILON, N. A. L.; ASSIS, G. B.; SOUZA, F. M.; BAITELLO, J. B. Plantas pequenas do cerrado: biodiversidade negligenciada. São Paulo, Editora Instituto Florestal. 2018.

SEIDL, C. Pesquisa de substâncias naturais inibidoras da acetilcolinesterase. Curitiba, PR. Dissertação de Mestrado. Universidade Federal do Paraná; 2010.

SILVA, H. R. L.; PEREIRA, P. S.; CARPINI, T. 2018. Caracterização de metabólitos secundários em qualea parviflora e rudgea viburnoides e atividade enzimática frente a acetilcolinesterase. Disponível em:

https://even3.blob.core.windows.net/anais/113478.pdf . Acesso em: 05/07/2019.

SILVA, L.S. Inibição de acetilcolinesterase e $\alpha$-amilase por extrato das folhas de mouriri elliptica martius. Rio Verde, GO. Dissertação de Mestrado. Instituto Federal Goiano; 2016.
SILVA, N.C.C.; FERNANDES JÚNIOR, A. Biological properties of medicinal plants: a review of their antimicrobial activity. Journal of Venomous Animals and Toxins including Tropical Diseases, v. 16, p.402-413, 2010.

SOUZA, P.M., Atividade de inibição enzimática por espécies vegetais do bioma Cerrado. Brasilia, DF. Dissertação de Mestrado. Universidade de Brasília; 2011.

TEIXEIRA, J. B.; SOUZA JUNIOR, P. R. B. ;HIGA, J.; THEME FILHA, M. M., Doença de Alzheimer: estudo da mortalidade no Brasil, 2000-2009. Cad. Saúde Pública, Rio de Janeiro, v.31, n.4, p.1-12, 2015.

VIEGAS JUNIOR, C. et al., Naturalv products as candidates for useful drugs in the treatment of Alzheimer's disease. Quim Nova. v.27, n.4, p. 655660, 2004.

VILA VERDE, G. M.; PAULA, J. R.; ARNEIRO, D. M. Levantamento etnobotânico das plantas medicinais do cerrado utilizadas pela população de MossâmedeGO. Revista Brasileira de Farmacognosia, v. 13, p. 64-66, 2003.

VINUTHA, B.; PRASHANTH D.; SALMA K.; SREEJA SL.; PRATITI D.; PADMAJA R.; RADHIKA S.; AMIT A.; VENKATESHWARLU K.; DEEPAK M. Screening of selected Indian medicinal plants for acetylcholinesterase inhibitory activity. Journal of Ethnopharmacology, v.109, p.359-63, 2007. 